\title{
HIGH GAIN ANTENNA CALIBRATION ON THREE SPACECRAFT
}

\author{
Joseph A. Hashmall \\ a.i.solutions, Inc., 10001 Derekwood Lane, Lanham, MD 20706, USA \\ joseph.hashmall@ai-solutions.com
}

\begin{abstract}
This paper describes the alignment calibration of spacecraft High Gain Antennas (HGAs) for three missions. For two of the missions (the Lunar Reconnaissance Orbiter and the Solar Dynamics Observatory) the calibration was performed on orbit. For the third mission (the Global Precipitation Measurement core satellite) ground simulation of the calibration was performed in a calibration feasibility study. These three satellites provide a range of calibration situations-Lunar orbit transmitting to a ground antenna for $L R O$, geosynchronous orbit transmitting to a ground antenna for SDO, and low Earth orbit transmitting to TDRS satellites for GPM. The calibration results depend strongly on the quality and quantity of calibration data. With insufficient data the calibration function may give erroneous solutions. Manual intervention in the calibration allowed reliable parameters to be generated for all three missions.
\end{abstract}

Keywords: High Gain Antenna, Calibration, SDO, LRO, GPM, Alignment

\section{Introduction}

The three missions discussed in this paper are the Lunar Reconnaissance Orbiter (LRO), the Solar Dynamics Observatory (SDO), and the Global Precipitation Measurement (GPM) core satellite. These three missions represent three very different situations for High Gain Antenna calibration. Calibration of each presented difficulties because of marginal data quantity or quality. Determination of the source of the calibration difficulties and relating these difficulties to data deficiencies illustrates the sensitivity of High Gain Antenna (HGA) calibration on data quantity and quality.

HGA calibration consists of finding values for the misalignments and biases of the antenna pointing mechanism that will maximize the received antenna signal power. Errors in the knowledge of these parameters result in HGA pointing directions that differ from those commanded and therefore reduce the received power. The only available measurement that can be used for this calibration is the radio frequency (RF) power measured by a receiver (usually reported as decibels of gain which is a logarithmic function of relative power). The basic method used for HGA calibration involves minimizing the differences between the measured and predicted power over observed HGA gimbal angles with respect to the calibration parameters.

An antenna je nninted by commanding its gimbals to desired angle values. The term "gimbal ang!coordinate space" refers to a 2-dimensional coordinate system in which the independent variables are the two gimbal angles. The gimbal angles change with time in order to point the HGA at a receiving antenna while both antennas are moving. The term "gimbal angle trajectory" is used here to mean the path of the commanded pointing direction in the gimbal angle coordinate system as a function of time.

Because the power at any pair of gimbal angles is a complex function of several independent calibration parameters, unambiguously finding the maximum power over the entire gimbal angle coordinate space requires accurate measurement of the power over a large portion of this space. For all of the missions describe here, marginal data quality or insufficient coverage of the gimbal angle coordinate space resulted in occasional ambiguous solutions. The correct solutions were successfully determined for each mission by examining the occurrence of different solutions, and manually determining which provided the best improvement in the antenna power. 


\section{High Gain Antenna Calibration}

\subsection{Parameterization}

The HGAs in this study are each controlled by a pair of gimbals. For generality these will be referred to as the inner gimbal (the gimbal attached to the spacecraft body) and the outer gimbal (the gimbal attached to the antenna dish). This arrangement is shown in Fig. 1.

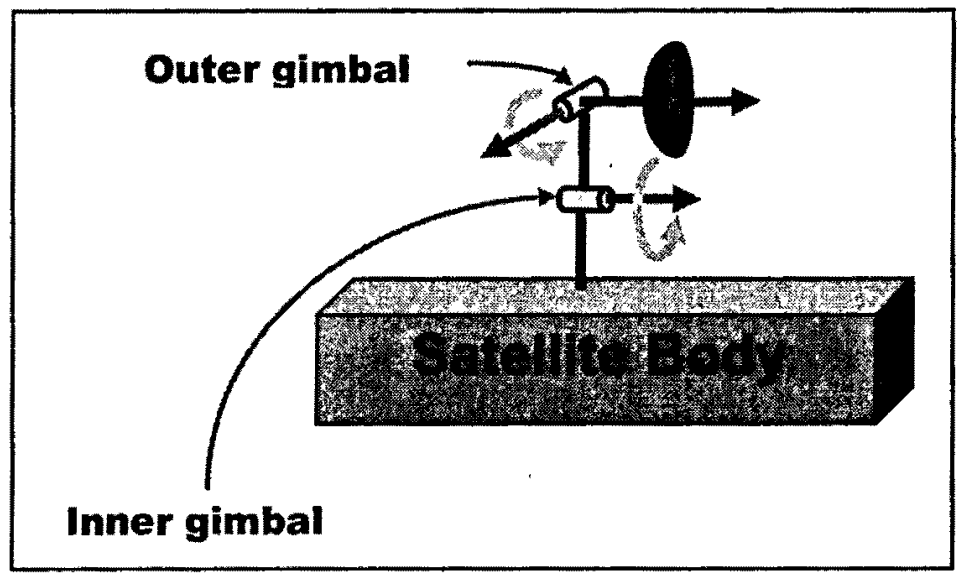

Figure 1. Typical Gimbal Geometry

The axes about which the gimbals rotate are mission dependent but, in order to provide pointing control, are generally perpendicular to each other. The two gimbals are often referred to as azimuth and elevation gimbals, but the correspondence between azimuth/elevation and inner/outer gimbals is different on different spacecraft. On LRO and GPM the inner gimbals were designated elevation gimbals and the outer designated azimuth gimbals. On SDO the situation was reversed.

In principal, the complete alignment parameterization of the HGA gimbal system would consist of the determination of 11 parameters representing angles (scale factors of the digital encoders were not considered):

- misalignment of the inner gimbal to the body ( 3 inner gimbal misalignment angles)

- misalignment of the outer to the inner gimbal ( 3 inner inter-gimbal misalignment angles)

- misalignment of the antenna dish to the outer gimbal ( 3 outer gimbal misalignment angles)

- 1 bias for each of the two gimbals.

The inner gimbal bias, one of the inner gimbal misalignment angles, and one of the inter-gimbal

" misalignment angies representrotations about the same axis ano can be combined. These combined parameters are represented by the inner gimbal bias.

Similarly, one of the inter-gimbal misalignment angles, the outer gimbal bias, and one of the outer gimbal misalignment angles can be combined and represented by the outer gimbal bias. In addition, the component of outer gimbal misalignment, which represents rotation about the dish central axis, is unobservable (because rotation about this axis causes very small changes in signal) and is not solved for.

The minimum parameter set that describes the HGA system misalignment consists of 2 inner gimbal misalignment angles, an inner gimbal bias, an inter-gimbal misalignment angle, an outer gimbal bias, and an outer gimbal misalignment angle. 


\subsection{Calibration}

The algorithm used for calibration has been previously described [1] but is summarized below.

The observations available for calibration are the gimbal angles and the power detected at a receiving antenna. For any observation set (gimbal angles and power) the antenna pointing direction is calculated using the spacecraft position and attitude and a set of calibration parameters. The angle between this pointing direction and the line-of-sight to the target is calculated and converted into a predicted relative power using the antenna's gain curve. The gain is scaled based on the distance from the transmitter to the target. If the values of the calibration parameters were optimal, the difference between the predicted gain and the observed power (as a gain) would approach zero.

The effect of compensation using any set of values for the parameters varies with the gimbal angles. In order to find a set of parameters that is valid over the entire range of gimbal angles the power must be sampled over a large portion of the gimbal angle coordinate space (i.e., the range of possible combinations of gimbal angles). These many sets of observations and derived gain differences must be combined into a least-squares loss function (Eq. 1) and that loss function minimized with respect to the calibration parameters:

$$
\text { Loss }=\left[\sum\left(G_{e x}-G_{o b s}\right)^{2}\right]^{1 / 2}
$$

where $G_{e x}$ is a function of the calibration parameters and depends on the position and attitude of the spacecraft and $G_{o b s}$ is the observed relative power represented as a gain. The loss function is calculated using the following steps.

1. Using the time of each power measurement and corresponding gimbal angles a line-of-sight vector is computed from the positions of the spacecraft and target antenna and converted into the body frame using the attitude.

2. The antenna pointing direction in the body frame is computed by rotating the dish axis vector (in the dish frame) through a series of coordinate transformations representing the nominal gimbal and dish alignments, the gimbal misalignments, the observed gimbal rotation angles, and the gimbal biases.

3. The angle between the line-of-sight vector and the antenna pointing direction is computed and the expected gain determined from a gain table (prelaunch gains measured at a series of angles from the dish axis).

4. The expected gain is compensated for the distance between the transmitting and receiving antennas.

5. The differences between measured and expected gains are used in Eq. 1 to determine the loss function.

Because the antenna pointing direction in step 3 is calculated using the calibration parameters the loss function depends on them. The MATLAB fminsearch function (an implementation of the simplex algorithm [2]) was used to determine the set of calibration parameters that minimizes the loss function value.

Previous analysis [3] has shown that parameter observability is improved if intentional, known, offsets of the pointing direction are commanded. To obtain the calibration data, the antennas were moved in a raster pattern centered on the expected target position during the periods in which the data was taken. 


\section{LRO Description and Results}

\subsection{LRO Overview}

The Lunar Reconnaissance Orbiter [4] was launched on June 18, 2009 and achieved Lunar insertion about five days later. It was placed in a nearly circular orbit around the moon with a period of about 2 hours at an altitude of about $50 \mathrm{~km}$. Its primary mission was to gather detailed data on the Lunar environment.

LRO was equipped with an HGA controlled by two mutually perpendicular gimbals, each having a pointing range of \pm 90 degrees. The inner gimbal and outer gimbals control elevation and azimuth, respectively. These gimbals were used to point the antenna at a dedicated 18 meter $\mathrm{Ka}$ Band antenna, located at White Sands New Mexico.

Because of the distance between the spacecraft and the ground station, the power received by the ground antenna is low and small errors in antenna pointing would have impeded communications. Concern with the power levels was a primary motivation of the decision to calibrate the LRO HGA. In addition, one of the payload instruments was attached to the antenna dish, therefore alignment of the HGA also aligned this instrument.

Because of the orbit geometry and the rotation of the Earth, only about five 50-minute contacts with the ground station were possible on any day. In order to track the ground station during these contacts, the gimbal angle trajectory follows nearly the full range in elevation ( -90 to +90 degrees) but with nearly constant azimuth gimbal angle. As the Moon rotates about the Earth, the azimuth gimbal angle in the trajectory gradually changes over its range, completing a cycle in one Lunar month. Obtaining data over a period of 2 weeks allows observations that nearly span the gimbal angle coordinate space.

The LRO HGA was successfully calibrated on orbit. The calibration details and results are given below.

\subsection{LRO Data}

The LRO data was taken from 11 contact periods of about 50 minutes each from day 181 through day 200 of 2009. Each contact period was on a separate day to provide the gimbal angle space coverage shown in Fig. 2.

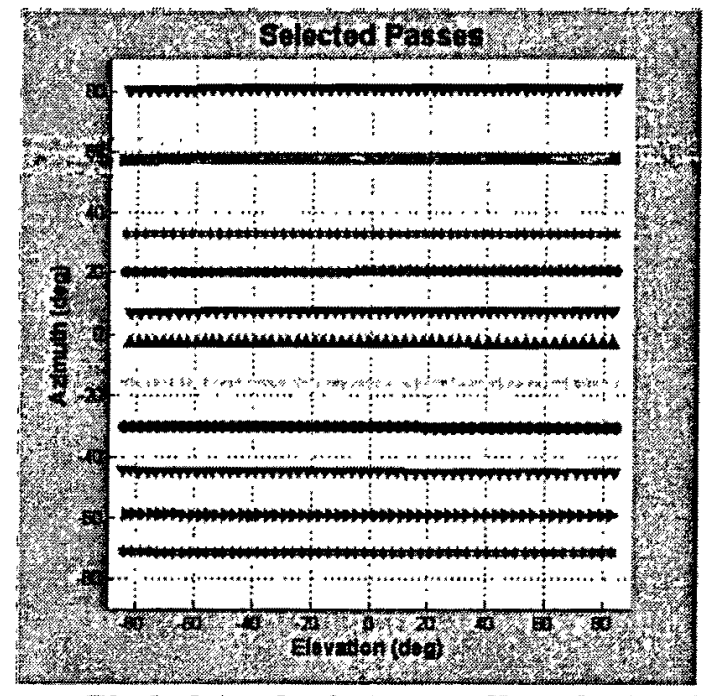

Figure 2. Target Gimbal Angles in Passes Used for LRO Calibration 
A raster pattern with an offset of up to \pm 0.5 degrees was applied to the required contact gimbal angles providing excellent observability.

Because of the distance between the spacecraft and ground, the power received at the dedicated ground antenna was low. There were also periodic signal dropouts during the calibration which were later correlated with wind at the ground antenna site.

A typical sample of receiver gain for LRO, before calibration parameters were uplinked, is shown in Fig. 3. This figure illustrates both the noise and the dropout periods. The power clearly varies as the gimbal angles are varied to track the ground antenna. This variation indicates that the pointing error varies with gimbal angle and that the calibration is poor. With ideal parameters, the gain would be nearly constant with gimbal angle (and therefore with time).

\subsection{LRO Results}

Calibration using the LRO data described above converged to reasonable values for the calibration parameters. However, in order to check the results the calibration was repeated several times with the same data but different initial estimates for the parameters. One of these attempts yielded parameters at convergence that were significantly different from the others. The existence of at least one least squares loss function local minimum corresponding to a suboptimum set of parameters was due to the relatively large noise on the data. These local minima had values of the loss function that were higher than the global minimum.

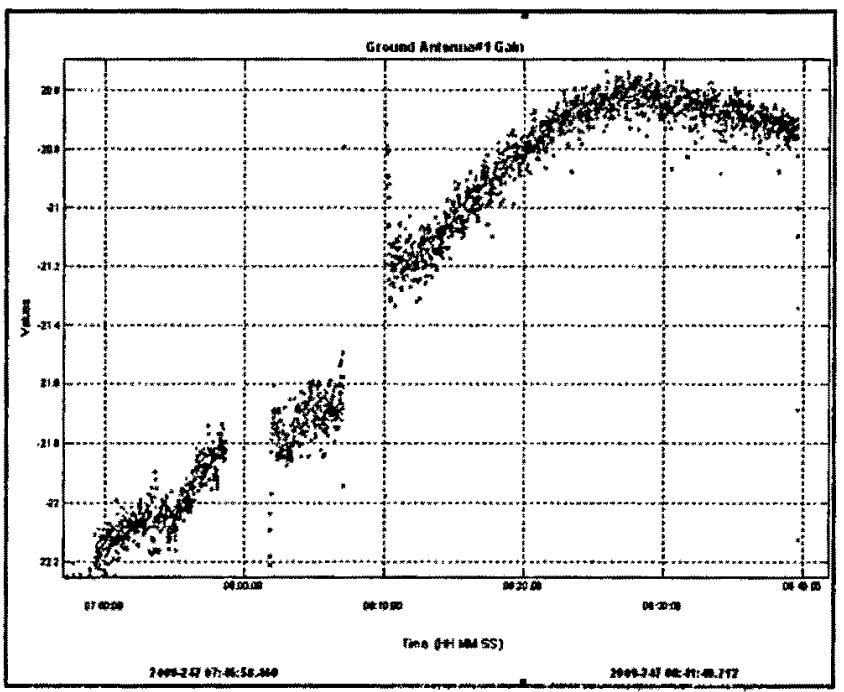

Figure 3. Observed Ground Antenna Gain for i LROFass Without Calibration Results

In order to determine the parameter set that provided a global minimum of the loss function and to investigate the differences in the results of calibrations with different starting estimates, the calibration was rerun 1000 times with randomly chosen initial parameter values, each of which had a uniform distribution of \pm 0.3 degrees. $95.5 \%$ of these attempts resulted in parameter sets with all values within 0.01 degrees of their mean values. Figure 4 shows a plot of the differences of parameters from their mean values in all 1000 parameter sets as a function of the loss function value. In this figure the 955 "similar" solutions (all parameter values within 0.01 degrees of their mean) are clustered under the dot plotted at zero angle difference and minimum loss function value. 


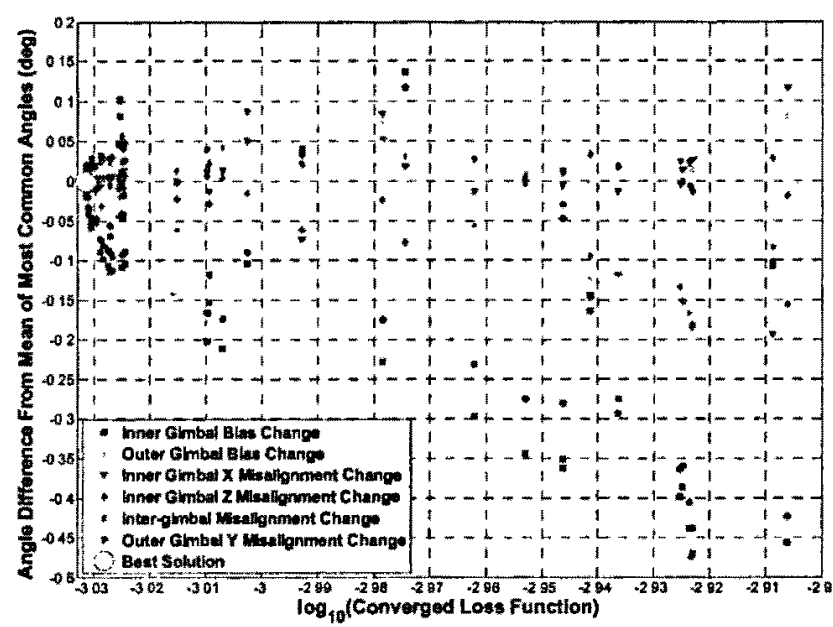

Figure 4. Parameter Differences From Mean of Most Common Solutions

The loss function value of these similar solutions was uniformly lower than that of the outlying solutions. Table 1 shows the mean of the "similar" solution values for LRO which was considered to be a reliable estimate of the parameters at the global minimum. (This table also shows the results for the two SDO antennas, discussed below).

Table 1. Final Calibration Results for LRO and Both SDO HGAs

\begin{tabular}{|l|c|c|c|}
\hline \multirow{2}{*}{ Calibration Parameter } & LRO & SDO $+Z$ & SDO -Z \\
\cline { 2 - 4 } & \multicolumn{3}{|c|}{ Value(degrees) } \\
\hline Inner gimbal misalignment 1 & 0.107 & 0.065 & 0.077 \\
\hline Inner gimbal misalignment 2 & 0.155 & -0.108 & -0.056 \\
\hline Inner gimbal bias & -0.033 & 0.186 & -0.411 \\
\hline Inter-gimbal misalignment angle & 0.159 & 0.040 & -0.018 \\
\hline Outer gimbal bias & 0.029 & 0.061 & -0.034 \\
\hline Outer gimbal misalignment angle & 0.190 & -0.004 & 0.003 \\
\hline
\end{tabular}

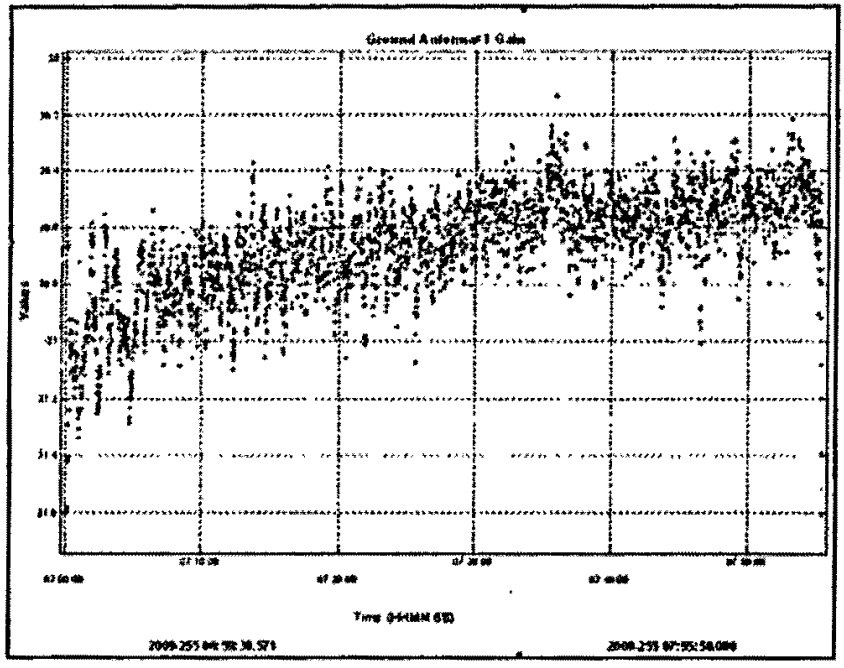

Figure 5. Observed Ground Antenna Gain for 1 LRO Pass With Calibration Results

The angles between pre- and postcalibration pointing directions as a function of the two gimbal angles is shown in Fig. 6. This figure show that the antenna pointing direction was improved by as much as 0.4 degrees at large azimuth angles but by much smaller amounts at small azimuth angles 
which is corroborated by the large improvement of the power at the start of a pass (large azimuth angles) as seen in Figs. 3 and 5.

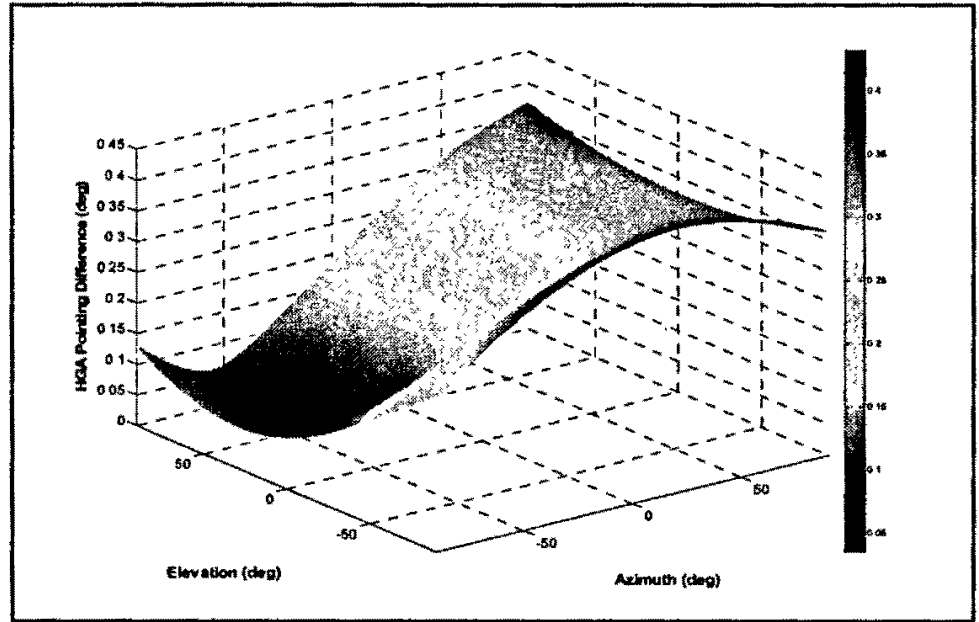

Figure 6. Change in Antenna Pointing Direction Due to LRO Calibration Parameters

\section{SDO Description and Results}

\subsection{SDO Overview}

The Solar Dynamics Observatory [5] was launched on Feb. 11, 2010 and achieved its mission orbit on March 16. Its orbit is geosynchronous at about $102 \mathrm{deg}$ west longitude and inclined at about 28.5 degrees. Its primary mission is to provide continuous, high resolution observations of the Sun. This mission requires nearly continuous transmission of a very high volume of data.

Prelaunch analysis and testing showed the possibility that the antenna deployment might be unrepeatable by as much as one degree. Concerns with the possibility of a large (one degree) misalignment and the critical requirement for uninterrupted communication were primary motivating factors in the decision to calibrate the SDO HGAs.

SDO was equipped with a pair of HGAs controlled by mutually perpendicular gimbals. The inner gimbal and outer gimbals control azimuth and elevation, respectively. The elevation gimbal is restricted to an angle range of about \pm 65 degrees (to prevent impingement of the radio frequency radiation on the spacecraft), but the azimuth gimbal has a full 360 degree rotation capability. Even with this restriction, there is an exclusion zone for each antenna that consists of a range of azimuth/elevation angles that must be avoided. The two HGAs were mounted on opposite sides of the spaxecraft and are referred to as the $+Z$ and $-Z$ antennas.

The SDO orbit provides continuous direct line-of-sight contact with a pair of dedicated ground antennas located at White Sands New Mexico. The ground antennas and the HGA dish are identical in design to those used by LRO. The received power was significantly greater than for LRO because of the smaller spacecraft to ground antenna distance.

Because of the orbit geometry, each SDO antenna follows nearly the same gimbal angle trajectory from orbit to orbit. Changes in path arise from the motion of the Earth about the Sun, and the complete range of gimbal angles is exercised only after a full year. In addition, during a large portion of the year (centered in the spring and autumn) both of the antennas have periods during each orbit in which their beams would impinge on the spacecraft. During these periods (called handover periods), the antenna that could impinge on the spacecraft is moved to a safe position and the other antenna is used for communication. These antenna handovers limit the portions of the gimbal angle trajectory that can be used for HGA calibration. 
Because of a very active commissioning schedule, efforts were made to reduce the time needed to generate the data needed to calibrate the SDO HGAs. As a result, the data provided was collected through less than a single gimbal angle trajectory. This data proved insufficient for automatically determining optimum calibration parameters. With manual intervention, consisting of determining subsets of the parameters and combining results, a reliable parameter set was found for each antenna and the results successfully used. The calibration details and results are given below.

\subsection{SDO Data}

HGA calibration for SDO provided difficulties different from those of LRO. The power levels were greater but the gimbal angle coordinate space coverage was much more limited. Initial pre-launch planning had assumed that each of the two antennas would provide 24-hours of data (one full orbit) so that a large portion of the gimbal angle coordinate space would be covered. The calibration data was accumulated during the mission commissioning phase and resources for HGA calibration had to compete with other requirements. In addition, SDO was in handover period which further limited the amount of data that could be used.

Figures 7 and 8 show the gimbal angle trajectory for the two antennas $(+Z$ and $-Z$ antennas) for the time in which the calibration data was generated. The large black areas near the top of the figures are the exclusion areas. Before the antennas are pointed to those regions, they must be moved to a safe position and the other antenna used for communication. Note that in these figures the evolution of gimbal angles with time (along the trajectories) moves to the right for the $+Z$-antenna and to the left for the $-\mathrm{Z}$-antenna. The green dots represent the times of antenna slews away from the exclusion region as well as start and end times of planned calibration data generation.

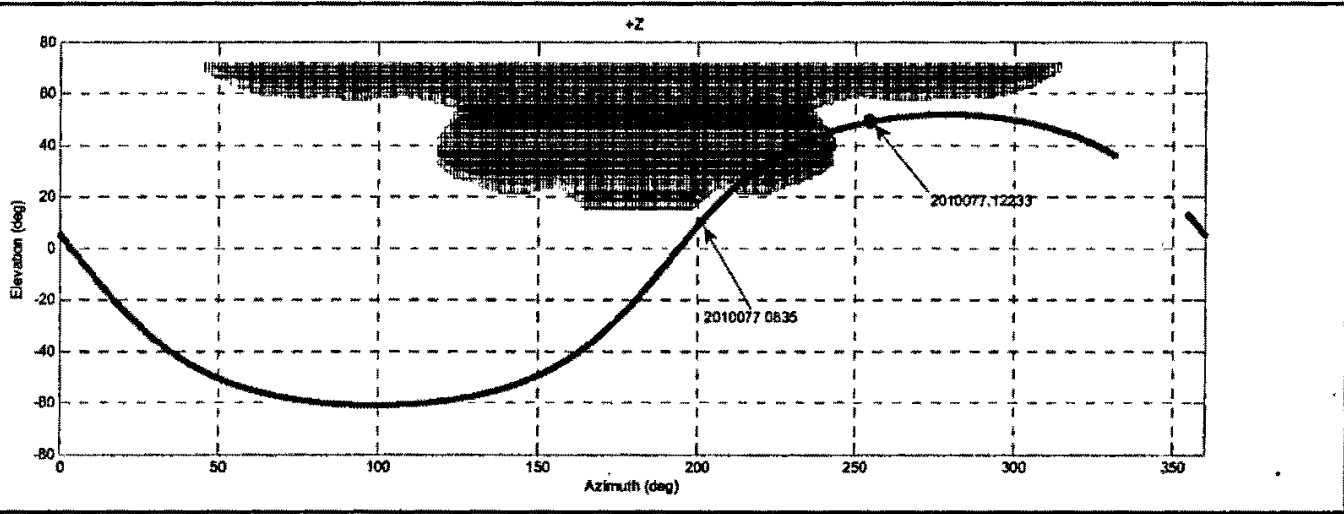

Figure 7. Gimbal Angle Trajectory for the SDO +Z Antenna

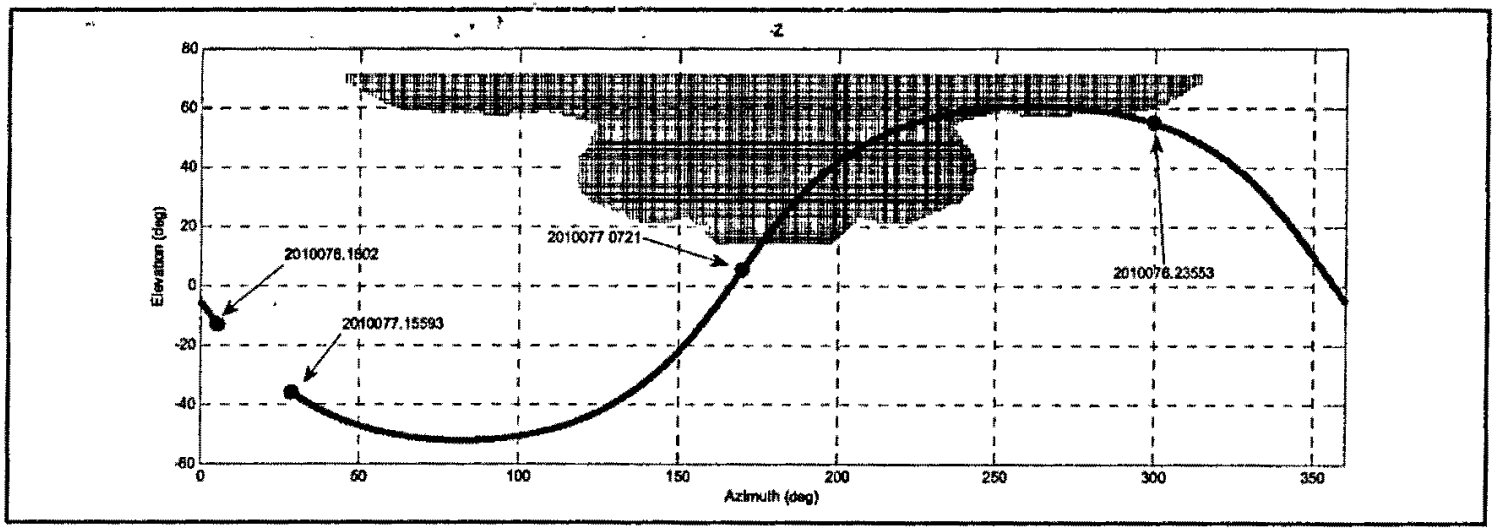

Figure 8. Gimbal Angle Trajectory for the SDO $-Z$ Antenna 
The initial data that was generated for calibration was limited to a period of about 13 hours for the $+Z$-antenna and less than 10 hours for the $-Z$-antenna. For both antennas the portion of the trajectory used was on the left sides of the trajectory plots. This data proved insufficient for calibration. Following the LRO experience, calibrations were attempted starting with different initial parameter estimates and the results differed significantly.

In order to improve the calibration, additional spans of data were recorded for each antenna. To avoid the exclusion areas, the spacecraft was rotated about the Sun line by -30 degrees, which had the effect of decreasing the elevation angles in the trajectory plots sufficiently to fall outside the exclusion zone at large azimuth angles. An additional eleven hours of observations for the $+Z$ antenna and twelve hours of observations for the $-Z$ antenna were collected, and calibration was performed using a combination of both sets of data for each antenna.

\subsection{SDO Results}

Before calibration was attempted, power was measured with a series of coarse offsets in pointing direction. This test showed large misalignments (several tenths of a degree) which caused loss of contact at some offsets. These misalignments were accounted for when calibration data was generated in order to prevent loss of contact.

It was difficult to achieve consistent results using the SDO data. Although the data trajectory spanned nearly the full range of gimbal angles, it did not cover a significant fraction of the gimbal angle space. Minimization of the loss function resulted in a wide variation in resulting parameter sets depending of the starting guesses due to the existence of numerous local minima. Because it was impractical to obtain additional data (covering a larger portion of the gimbal angle space would have required either waiting months for the orbit to shift or executing large maneuvers), a manual procedure was established that led to consistent, reliable values for all calibration parameters at the global minimum.

The manual procedure consisted of three steps. It was found that the loss function was most sensitive to variations in the two biases and one inner gimbal misalignment. In the first step, these parameters were determined while keeping the other parameters at nominal values. The second step applied the values determined in the first step as constants and solved for the other three parameters. The third step, starting with the values determined for all of the parameters in the first two steps, solved for all of the parameters.

The results of this three-step calibration were consistent-the final values were essentially unchanged regardless of the initial starting parameters. Table 2 (above) includes the final values of the calibration parameters. Figure 9 shows the power error (expected-observed) for both antennas as a function of time for one of the caliuration intervais on an arbitrary scale.

Since these are power errors, perfect calibration parameters would result in values of 0 across the entire time span. The oscillations in the values are due to the raster scan. It is clear that the power errors after application of the parameters (black curve) is much closer to 0 error and shows less variation with time than do the precalibration values (red curve). 


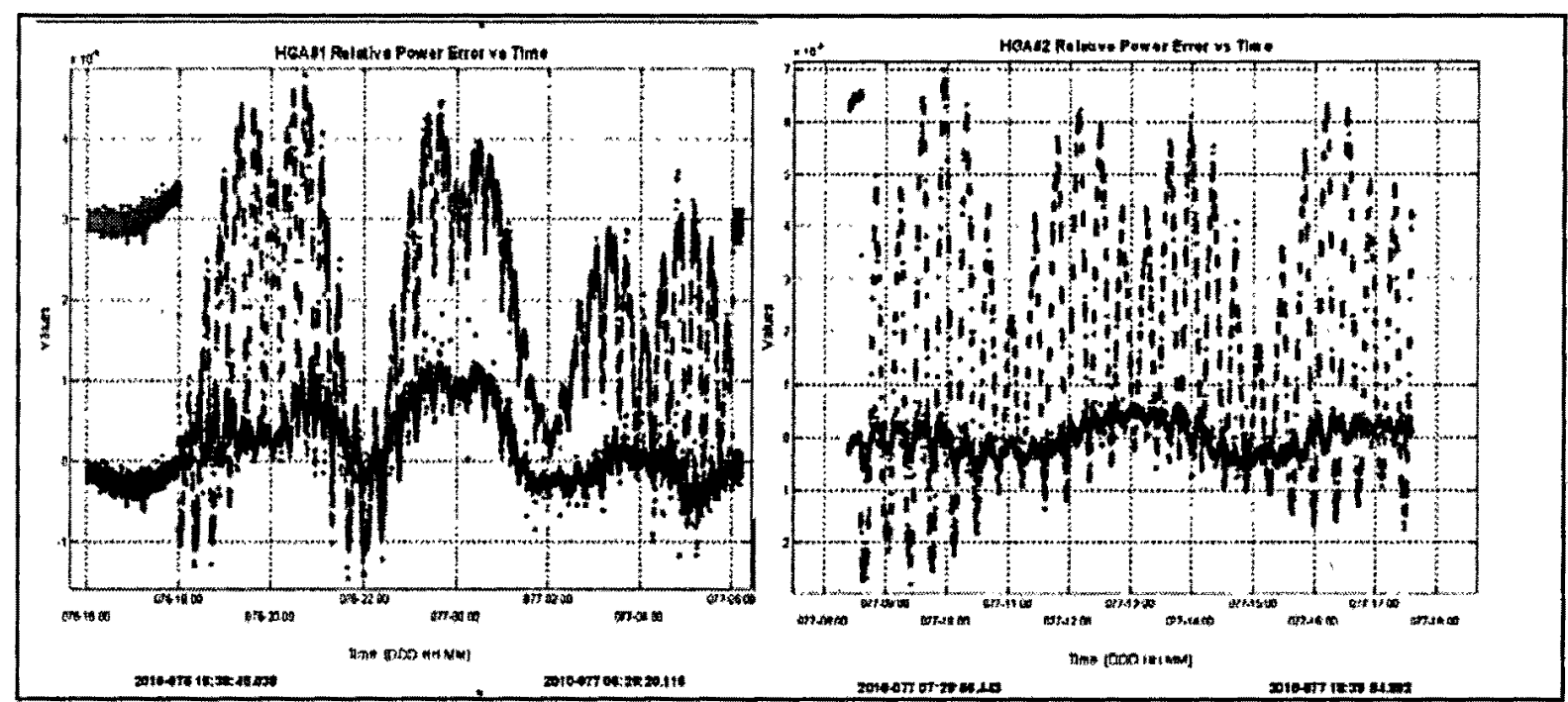

Figure 9. Effect of Applying Calibration Parameters on Power Error for Both SDO HGAs.

(Precalibration errors are shown in red, postcalibration errors are shown in black).

Figure 10 shows how the application of the final calibration changed the pointing direction of the two SDO antennas. The angles between the pointing directions before and after application of the calibration parameters are shown. The $-\mathrm{Z}$ antenna pointing direction was improved by as much as 0.2 degrees while that of the $+Z$ antenna was improved by about half that value.

The great difficulty in calibrating the two SDO antennas was due to the poor coverage of the gimbal angle coordinate space. The coarse corrections initially applied proved sufficient for operations. Tables based on the additional calculated parameters were generated and if antenna power becomes a problem, could be uplinked when needed.

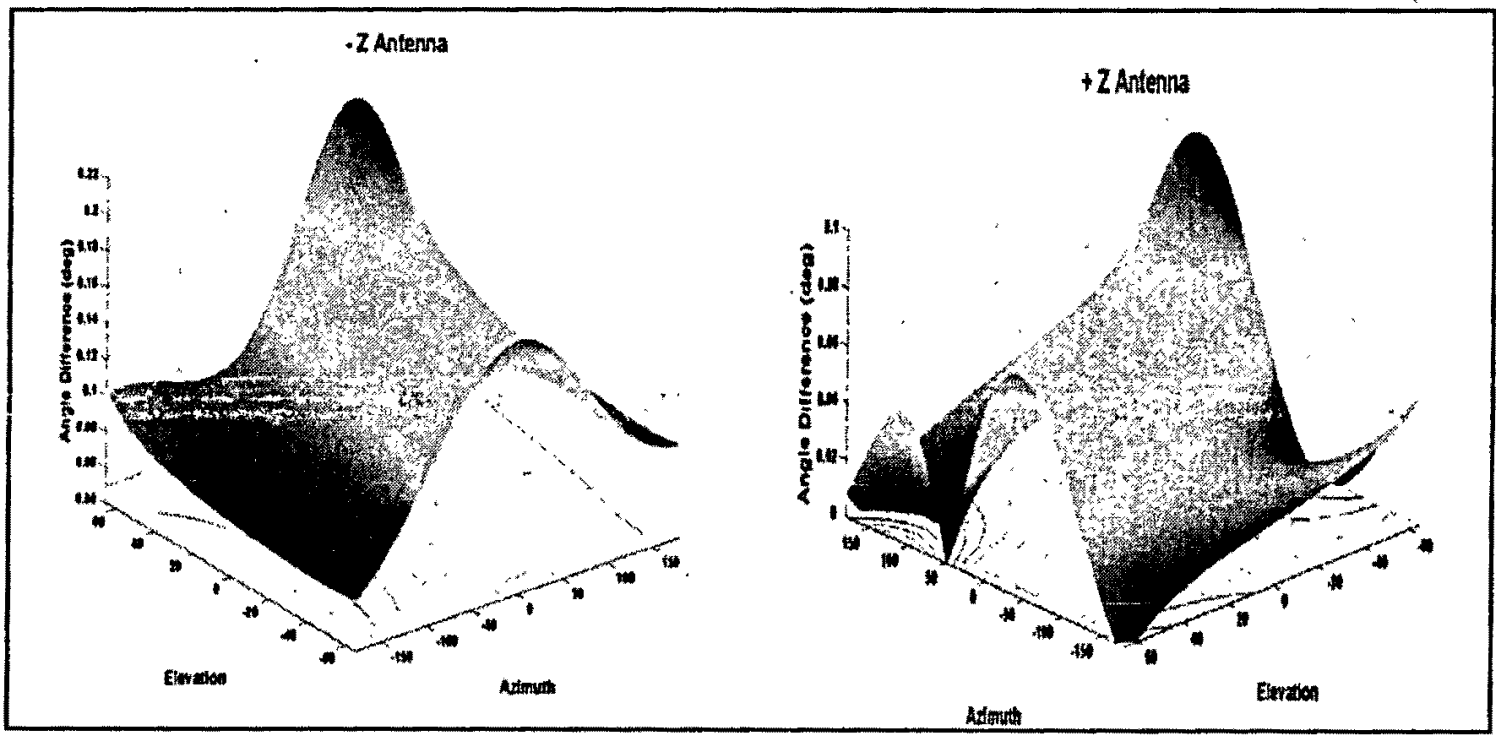

Figure 10. Effect of Calibration Parameters on Pointing Directions for Both SDO HGAs 


\section{GPM Description and Results}

\subsection{GPM Overview}

The Global Precipitation Measurement [6] mission's core satellite is scheduled to be launched in July 2013 into a near-circular, low-earth orbit (about $400 \mathrm{~km}$ altitude), with an inclination of about 65 degrees. It is a joint NASA/JAXA mission that will replace and expand on the capabilities of the Tropical Rainfall Measurement Mission (TRMM). Its primary mission is to measure and characterize worldwide precipitation. It is required to report data soon after it is measured so it needs the capability of near continuous communications.

The GPM core satellite is equipped with a single HGA, controlled by two mutually perpendicular gimbals, each having a pointing range of \pm 90 degrees. The inner gimbal and outer gimbals control elevation and azimuth, respectively. The gimbal system is similar in design to LRO's. The antenna is designed to communicate through the Tracking and Data Relay Satellite System (TDRSS). For the LRO and SDO missions the power was measured at the ground antenna. For GPM the power transmitted by the TDRSS satellites was to have been measured on the GPM satellite. Fortunately, the function describing the power change as a function of the calibration parameters is the same regardless of whether the transmitting antenna (mission HGAs for LRO and SDO, TDRS antenna for GPM) or the receiving antenna (ground antennas for LRO and SDO, mission HGA for GPM) is calibrated. Therefore, the same algorithm that was used for LRO and SDO could be used all three missions.

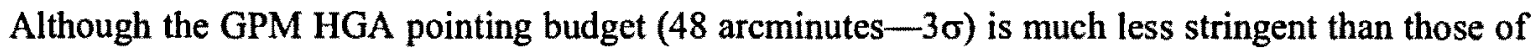
LRO and SDO, structural analysis showed the possibility of antenna pointing errors larger than these requirements due to large vibrations (jitter) of the antenna structure. One proposed mitigation of the pointing errors was antenna calibration, so a study was performed to evaluate feasibility and probable results of such a calibration. The study showed that the antenna pointing errors could be sufficiently reduced to satisfy mission requirements only if sufficient TDRSS contacts were allowed to provide sufficient coverage of the gimbal angle coordinate space. Subsequent more exact structural analysis showed much smaller vibrations, removing the need for HGA calibration. A description and the results of the feasibility study are given below.

\subsection{GPM Simulation}

For GPM the situation differed from that of the other two spacecraft. The transmitter was to be a TDRS and the receiver the HGA on GPM. The mathematical description of the problem was identical but the signal was expected to be weak due to the small size of the receiver dish.

The major contributor to signal noise was expected to be pointing variations due to vibrations of the antenna structure. These were predicted to have a frequency of approximately $9 \mathrm{~Hz}$ with amplitude such that the standard deviation of the angle errors was 10 arcminutes $(1 \sigma)$

A simple simulator was created to simulate the received HGA's power given a set of misalignments and gimbal angles. The gimbal angles were computed as those necessary to point the antenna at a TDRS during specified periods plus specified offset raster scan angles. The simulator used realistic ephemerides for both GPM and TDRS and represented the noise using the predicted antenna vibrations to model the antenna pointing errors.

The feasibility study investigated the calibration results as a function of the amount of TDRS contact provided for calibration. Two contact strategies differed in the gimbal angle range and quantity of the TDRS contacts. In both strategies a single TDRS was used. If neither proved successful, additional data could have been simulated through use of additional TDRSs. Contacts 
with multiple TDRSs would probably be an advantage, but the relative transmission power of the different TDRSs would have to be determined and compensated.

For both strategies, the times at which the TDRS was visible were computed for a 24 hour period. In strategy 1 , contact was assumed during the 10 minute period at the center of each interval during which TDRS was visible. This resulted in a restricted range of gimbal angles during the contact. In strategy 2, two additional 10 minute periods were selected - the first starting 5 minutes after TDRS was first visible and the second ending 5 minutes before the end of TDRS visibility. This second strategy provides observations over a larger range of gimbal angles.

The gimbal angle trajectories for a single day of simulated data are shown in Fig. 11.

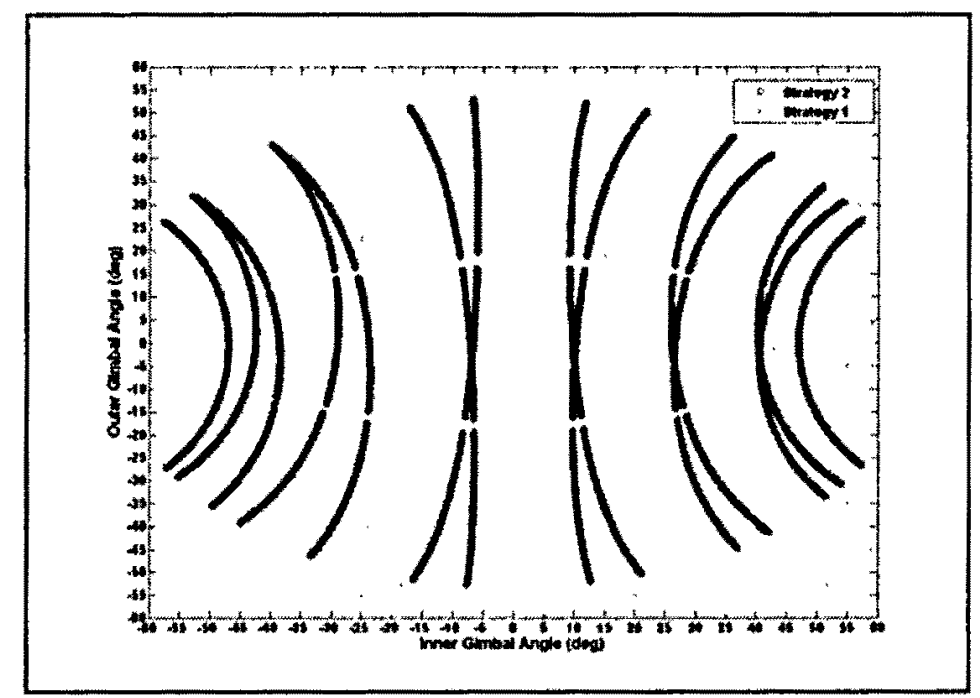

Figure 11. Nominal Gimbal Angles Necessary for TDRS Contact Shown for the Two Strategies. (Strategy 1 trajectory shown in blue, strategy 2 trajectory shown in red).

Power was simulated at gimbal angles along the trajectories by computing the angle between pointing directions with and without using randomly selected calibration parameters and converting these angles into observed gains by reversing the procedure described in section 2.2 above. Calibration solutions were found using 1000 randomly selected sets of "truth" misalignments and biases with a range of \pm 0.5 degrees.

\subsection{GPM Results}

Since the parameters used to create the simulated data were available (the "truth" parameters), the accuracy of the calibration could be evaluated over the entire gimbal angle coordinate space by comparing the pointing directions at any gimbal angles using the "truth" parameters with those using the solution parameters. The angle differences are pointing errors since the true simulated pointing directions are known.

The gimbal angle coordinate space spans the range of \pm 90 degrees for both gimbals. Antenna pointing angle errors using gimbal angle values at 1 degree intervals throughout this range were evaluated and the standard deviation of the errors for the $\mathbf{3 2 7 6 1}$ angle combinations were used as a measure of the overall calibration error. These standard deviations for all 1000 smaples of parameters are shown in Fig. 12.

With strategy $1,77 \%$ of the cases are met the pointing accuracy requirement (16 arcminutes $1 \sigma$ as indicated by the dashed line in Fig. 12). With strategy $2,99.8 \%$ of the cases met requirements. 


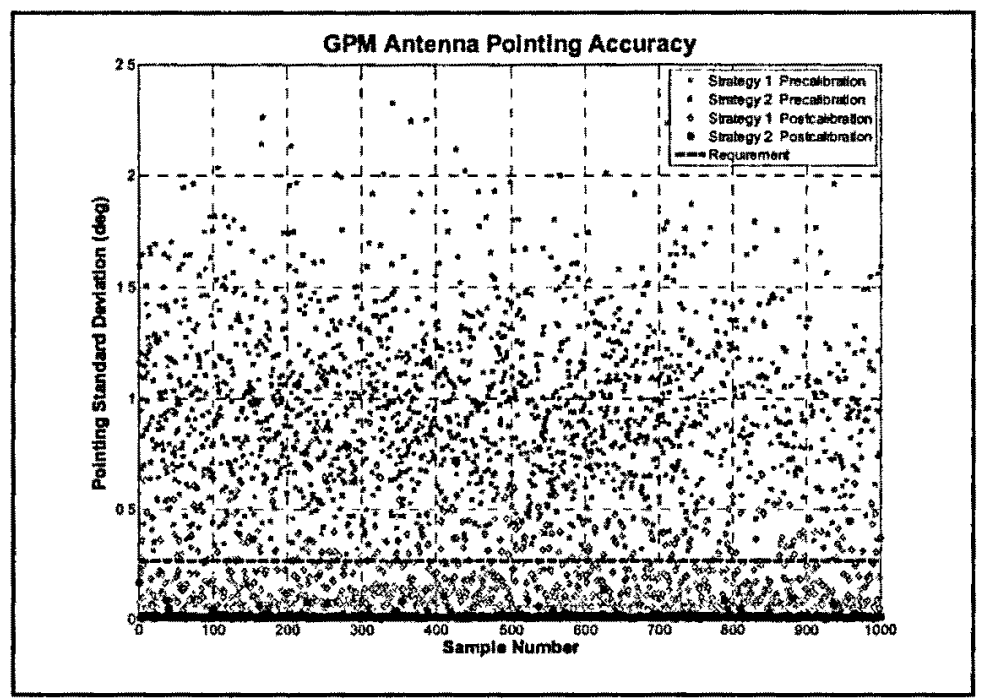

Figure 12. GPM Pre- and Post-calibration Pointing Uncertainties (1б) Using Two TDRS Contact Strategies

The much poorer calibration accuracy shown for the strategy 1 case, results from the smaller portion of the gimbal angle coordinate space covered. In strategy 1, the outer gimbal angle is exercised over a range of less than $\pm 20 \mathrm{deg}$. Over this range, the effect of the inner gimbal bias is strongly correlated with that of the dish-to-outer gimbal misalignment. The values of these parameters in the solution are therefore not independently unobservable; large errors in the estimated value of one can cancel the effect of errors in the other.

When the pointing errors are determined over a larger portion of the gimbal angle coordinate space, the cancellation of the effects of these two parameters no longer applies and the calibration produces much smaller pointing errors. This is evident by examination of Figs. 13-15. Figure 13 shows an example of pointing errors over the entire range of gimbal angles for a typical case before calibration. Note that the pointing errors nearly 2 degrees-probably larger than would occur in the actual mission-which may indicate that the simulated calibration parameter errors have larger values than is realistic. Figure 14 shows the pointing errors for the same case using data from strategy 1. Although the maximum pointing error has been reduced to about 0.25 degrees, there is still a regular, significant pattern of large values at elevation angles near their extremes $( \pm 90$ degrees). The symmetry of the errors as a function of gimbal angles is due to the correlation between the inner gimbal (elevation) bias and the dish-to-outer gimbal misalignment. Figure 15 shows results for the same case using data from strategy 2. The addition of data over a larger portinn of the gimbai angle coordinate space breaks the correlation and aliows a solution to be determined with maximum errors of less than 0.03 degrees.

\section{Conclusions}

Clearly, the success of HGA calibration is strongly dependent on the quantity and quality of the calibration data.

For LRO, the gimbal angle coordinate space was well covered, but the data had poor signal-to-noise resulting in local minima in the loss function. A larger data volume would have minimized the problem. Lacking the additional data a statistical method (solving for the parameters repeatedly with many initial guesses) was used instead. This statistical method led to a reliable set of parameters which, when used on board, improved the measured power. 


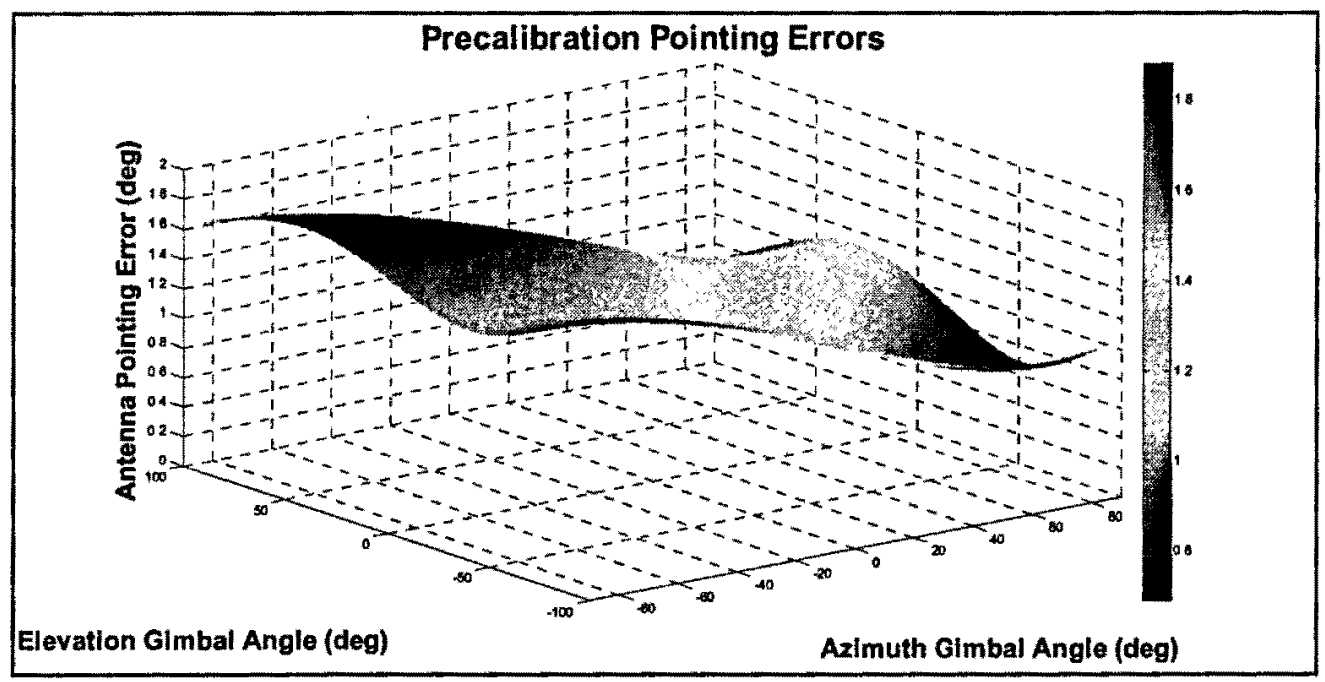

Figure 13. Example of GPM Precalibration Pointing Errors

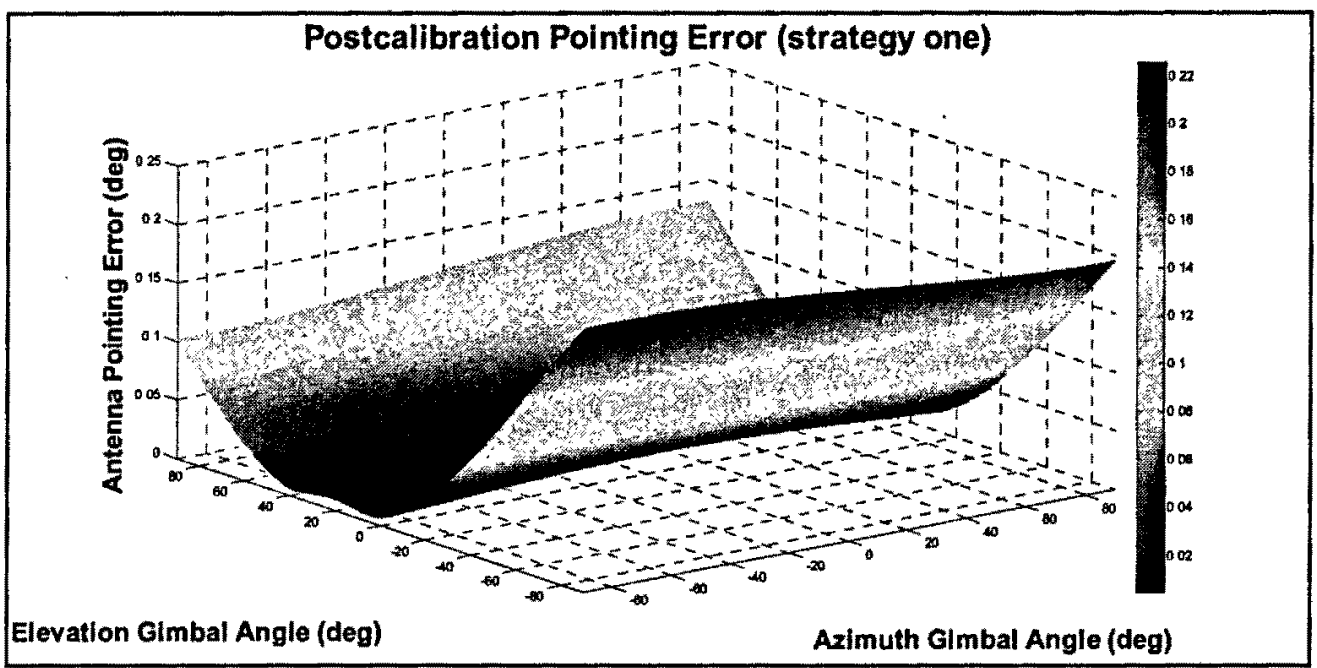

Figure 14. Example of Postcalibration Pointing Errors Using Strategy 1

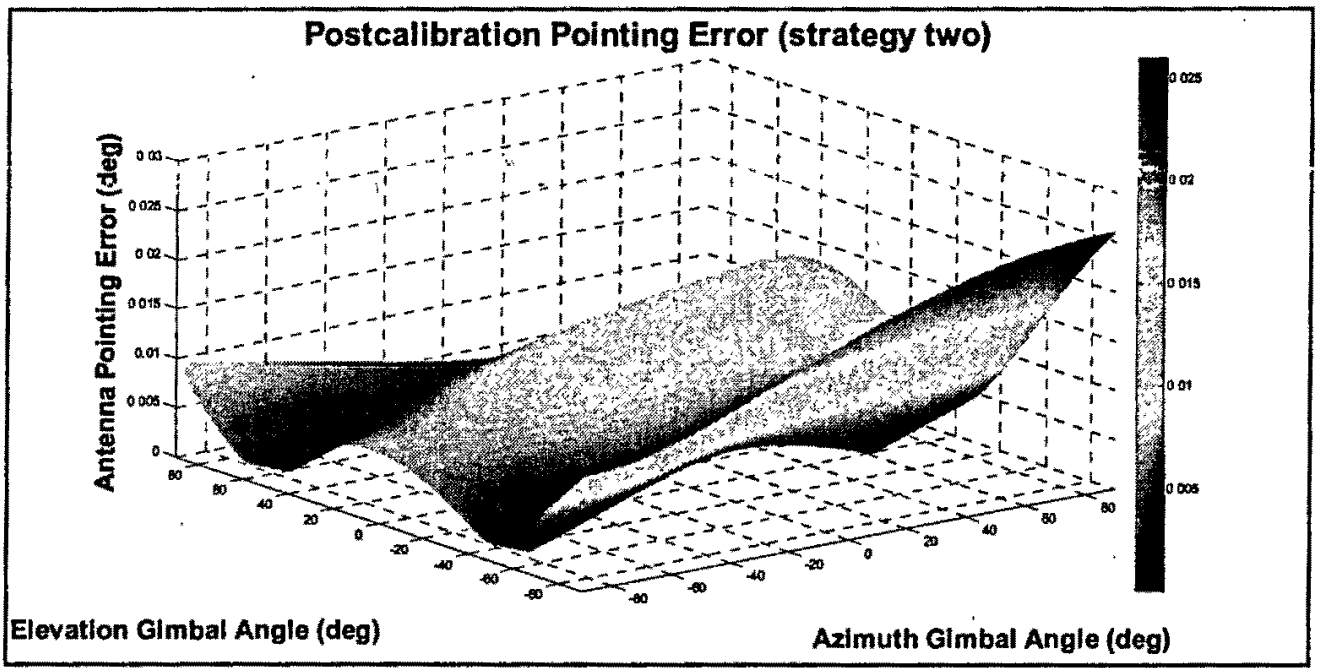

Figure 15. Example of Postcalibration Pointing Errors Using Strategy 2 
For SDO, the signal-to-noise ratio was satisfactory but the coverage of the gimbal angle coordinate space was poor. In order to obtain reliable parameters, additional data was obtained. Even using the additional data a step-wise manual calibration was needed to obtain consistent reliable parameters. The final calibration parameters were validated and are available to improve antenna power if necessary.

For GPM, simulations showed the ability to perform an HGA calibration with high probability of success using contacts with a single TDRS. The study clearly showed that if only a limited portion of the gimbal angle coordinate space calibration is used, parameters may be strongly correlated and calibration unreliable. However, if an appropriate set of TDRS contacts were selected calibration of the GPM antenna is completely feasible. Although GPM does not presently plan to perform a calibration, the algorithms and software used for SDO and LRO have been shown to work for GPM, and the calibration software is available if on orbit experience causes reconsideration of calibration.

Fortunately, for the three missions described here flaws in the quantity and quality of the data were overcome and reliable results were obtained. The primary conclusion of this study is that accurate, consistent High Gain Antenna calibration requires large amounts of data covering most of the gimbal angle coordinate space. Missions requiring High Gain Antenna calibration must plan to obtain this data.

\section{Acknowledgments}

This work was supported by the National Aeronautics and Space Administration (NASA)/Goddard Space Flight Center (GSFC), Greenbelt, MD, USA, under the Flight Dynamics Support Services (FDSS) contract: NNG10CP02C. The support of Philip Calhoun and Robert DeFazio of NASA Goddard Space Flight Center is greatly appreciated.

\section{References}

[1] Hashmall, J. A.," "Calibration Of Gimbaled Platforms: The Solar Dynamics Observatory High Gain Antennas", Proceedings of the $19^{\text {th }}$ International Symposium on Spaceflight Dynamics, ISTS2006-d-36, Kanazawa, Japan, 2006

[2] Lagarias, J. C., Reeds, J. A., Wright, M. H., and Wright, P. E., "Convergence Properties of the Nelder-Mead Simplex Method in Low Dimensions," SIAM Journal of Optimization, Vol. 9, Number 1, 1998.

[3] Hashmall, J. A., "Solar Dynamics Observatory (SDO) High Gain Antenna (HGA) Calibration Analysis", MOMS Report FDF-85-003, NASA Goddard Space Flight Center, February 2005.

[4] Sedlak, J.E. and Houghton, M. B., "Lunar Reconnaissance Orbiter (LRO) Attitude Maneuver Planning," Proceedings of the $21^{\text {st }}$ International Symposium on Spaceflight Dynamics, CNES, Toulouse, France, 2009.

[5] Natanson, G., Mason, P. Bourkland, K., Vess, M., Hashmall, J., Shulman, S. Ottenstein, N., Starin, S., McIntosh, R., DeFazio, R., "Solar Dynamics Observatory (SDO) Ascent Planning and Momentum", AIAA-2010-2354, Proceedings of the SpaceOps 2010 Conference, Huntsville, Alabama, USA, 2010

[6] NASA Goddard Space Flight Center web site: http://gpm.gsfc.nasa.gov/ 